BENM 2021

International Scientific and Practical Conference "Biotechnology, Ecology, Nature Management"

\title{
REGENERATION OF CAUDAL FIN THE INFLUENCE OF THE FOOD ADDITIVE «CHIGYANY ROZ»
}

\author{
V. A. Purtskhvanidze (a)*, S. V. Smorodinskaya (b), Yu. G. Simakov (c), \\ M. V. Medyankina (d) \\ *Corresponding author
}

(a) K.G. Razumovsky Moscow State University of Technologies and Management (the First Cossack University), 73, Zemlyanoy Val str., Moscow, Russia, violetta@laservita.ru

(b) K.G. Razumovsky Moscow State University of Technologies and Management (the First Cossack University), 73, Zemlyanoy Val str., Moscow, Russia

(c) K.G. Razumovsky Moscow State University of Technologies and Management (the First Cossack University), 73, Zemlyanoy Val str., Moscow, Russia

(d) K.G. Razumovsky Moscow State University of Technologies and Management (the First Cossack University), 73, Zemlyanoy Val str., Moscow, Russia, m.medyankina@mgutm.ru

\begin{abstract}
Regeneration of the upper lobe of the caudal fin in juvenile Danio rerio fish after experimental amputation under the toxic effect of potassium dichromate and the addition of fermented milk product "Chigyany ROZ" to granulated fish feed was studied. The obtained results allowed us to establish that the product "Chigyany ROZ" has protective properties and protects the regenerating organ from the effects of the gnotoxicant. Regeneration of the zebrafish fin blade under study in a toxicant solution without adding Chigyany ROZ to the fish feed was suppressed, and on day 9 of the experiment it was approximately 2 times behind regeneration $\mathrm{b}$ compared to the control. Luminescent analysis of the regenerating fin blade under a microscope, after staining with acridine orange, he showed that fish fed with addition of the lactic acid product "Chigyany ROZ" in blastem contained more RNA, which apparently contributes to the regeneration of the amputated zebrafish fin blade.
\end{abstract}

2672-8575 @ 2022 Published by European Publisher.

Keywords: A product of "Chigany ROZ", blastema, RNA, regeneration, upper blade. caudal fin. potassium dichromate 


\section{Introduction}

The caudal fin of the zebrafish (Danio rerio) is an excellent model for studying the regeneration of organs and tissues in vertebrates under the conditions of stimulating and antimutagenic factors. In this paper, we investigated the presence of the fermented milk dry skimmed product "Chigyany ROZ" with fruit and vegetable filler stimulating and protective effect during regeneration after removal of the upper lobe of the caudal fin in juvenile zebrafish. Currently, studies on caudal fin regeneration in zebrafish are becoming classic and are being conducted by geneticists, cytologists and developmental biologists. (Ishida et al., 2010; Kachanov et al., 2020; Niethammer et al., 2009; Traver et al., 2004). Reactivation of silent embryonic genes may play an important role in the mechanism of fin regeneration. This reactivation of genes may be associated with demethylation of histone H3. This is evidenced by the inhibition of the caudal fin regeneration process when the synthesis of me3K27 H3 demethylase (me3K27 $\mathrm{H} 3$ demethylase) is suppressed, since the promoter of one of the targets of this enzyme (dlx4a, a homeobox gene from the family of genes involved in the formation of appendages during embryogenesis) is not activated (González-Rosa et al., 2017; Kettleborough et al., 2013; Nikiforov-Nikishin et al., 2021).

It is quite clear that DNA and RNA play an important role in caudal fin regeneration, so researchers need to identify the localization of nucleic acids in the blastem. Cytochemical and histochemical methods for determining DNA and RNA in regenerating tissues rather than biochemical ones are best suited for detecting the distribution topology of nucleic acids (Kettleborough et al., 2013).

Zebrafish can regenerate various organs and tissues, including the heart, eyes, and fins. At the same time, regeneration takes place relatively quickly, and in just two weeks, almost complete restoration of tissues and organs is observed, including new bones, nerves, blood vessels, connective tissue and skin (González-Rosa et al., 2017).

Biologists are constantly discovering the new role of microRNAs in the body and believe that they affect cell division and differentiation, as well as their death. Numerous experiments conducted by American researchers with striped zebrafish allowed Poss and his colleagues to establish that the successful regeneration of severed fins is affected by the concentration of miR-133 microRNA in them. microRNAs regulate the expression of more than $30 \%$ of the genes encoding the structure of proteins. To date, about 5 thousand microRNAs have been identified in databases. Most (61\%) miRNA genes are located in the intron regions of protein-coding genes; however, miRNA genes can be localized in exon regions or intergenic regions. microRNAs are transcribed from genes called microRNA genes by RNA polymerase II (pol II), some by pol III (Jopling et al., 2010). In the damaged fin, the concentration of microRNA drops sharply, which triggers a chain of certain genes, as a result of which a growth factor is produced, and the fin regenerates.

If the level of miR-133 in the partially severed fin remained the same as in the surviving fin, then regeneration is very weak or stops altogether. A drop in the microRNA concentration signals cells to divide. Therefore, this molecule protects the organ from uncontrolled cell proliferation. When an organ is damaged, when part of it is cut off, the concentration of micro-RNA drops and encourages the regenerating tissue, and if necessary, the entire organ, to regenerate. 
Researchers of this process believe that a similar system of gene regulation of microRNA synthesis, which encourages tissues and organs to recover, has also been preserved in mammals, including humans, but it has been repressed for some reason (Belyaeva et al., 2010; Bradford et al., 2017; Howe et al., 2013).

\section{Purpose of the Study}

In this study, we applied luminescent cytochemical analysis to detect the localization of DNA and RNA in the regenerating fin of fish. It is quite clear that in this way it is possible to identify all types of nucleic acids contained in the regenerating organ. However, a sharp decrease in red fluorescence in the regenerating fin blastem is likely to indicate a decrease in microRNA content. Thus, it becomes possible to visualize the topology of RNA distribution, among which microRNA is also detected, and determine whether Chigana ROZ has the ability to stimulate regeneration. The aim is to determine whether the studied product has protective properties for regeneration in the presence of a genotoxicant in the aquatic environment, in particular hexavalent chromium, which suppresses the processes of organ regeneration and cell proliferation (Smorodinskaya \& Simaov, 2019).

\section{Research Methods}

Experiments on the regeneration of the upper part of the caudal fin in Danio rerio with the addition of the studied product "Chigyany ROZ" to the feed were carried out in sewage tap water and at active concentrations of potassium dichromate on juvenile fish. Fish at the age of 2 months, $2 \mathrm{~cm}$ long, were placed in plastic aquariums of 12 individuals in sewage tap water. The volume of water in each aquarium was 2 liters. In total, only 12 aquariums were delivered to conduct experiments in three replicates. Of these, three aquariums with fish in sewage tap water without the use of "Chigany ROZ" (control). Regeneration of the cupped caudal fin blade in zebrafish was studied in three aquariums, which were fed with calculated doses of "Chigyany ROZ". In the following variants, three aquariums with fish were supplied, in which regeneration of the upper part of the fin in zebrafish was studied in solutions of potassium dichromate at a concentration of $0.5 \mathrm{mg} / 1$, but without adding the product "Chigyany ROZ" to the feed. Finally, the last three aquariums were filled with fish that simultaneously removed the caudal fin blade and received a supplement of the product "Chigyany ROZ" with food. We have previously shown that the potassium dichromate concentration of $0.5 \mathrm{mg} / 1$ is effective and suppresses caudal fin regeneration in zebrafish (Aleksandrov \& Simakov, 2013). Fish were fed with French food "Esturgeon" with a granule size of 200-300 microns. The fish were adapted to the new conditions for a week before the experiment.

The experiment was carried out on fish in which the upper part of the homocercal caudal fin was cut off with a sharp-edged tubular punch with a diameter of $2 \mathrm{~mm}$. The operation takes a few seconds, and the fish do not suffer from a lack of oxygen. Young zebrafish were then placed in aquariums. Fish do not directly take fractions of "Chigany ROZ" tablets, so the test product was added to fish feed by weak pressing into the granules in a porcelain mortar with a pestle. "Chigyany ROZ" was added at the rate of 
0.5 tablets of "Chigyany ROZ" per $200 \mathrm{~g}$ of live weight of fish. The duration of the experiment was 9 days. Under the control of MBS-10, fish were viewed and photographed after 3, 6, and 9 days.

For luminescent analysis, one individual was selected from each aquarium after 3 and 9 days (three for each experimental variant), which were clogged after euthanasia with ether, their caudal fin was cut off, total histological preparations were prepared, and after staining, the fin was photographed with a digital camera under a $3 \mathrm{~L}$ MB luminescent microscope. For this purpose, the caudal fin was stained with acridine orange, which makes it possible to detect the localization of DNA and RNA (Karnaukhov, 2002) in the regenerating and intact fin lobe under fluorescence. The scheme of setting up the experiment in one repetition is shown in Figure 1.

\begin{tabular}{|c|c|c|c|}
\hline $\begin{array}{l}\text { Sewage tap water } \\
\text { for } 12 \text { zebrafish with } \\
\text { the upper part of the } \\
\text { caudal fin cut off } \\
\text { Feed without the } \\
\text { product "Chigyany } \\
\text { ROZ" }\end{array}$ & $\begin{array}{l}\text { Sewage tap water } \\
\text { for } 12 \text { zebrafish with } \\
\text { the upper part of the } \\
\text { caudal fin cut off } \\
\text { Food with the } \\
\text { product "Chigyany } \\
\text { ROZ" }\end{array}$ & $\begin{array}{l}\text { Potassium dichromate } \\
\text { solution conc. } 0.5 \mathrm{mg} \\
\quad / 1 \text { of } 12 \text { zebrafish } \\
\text { with the upper part of } \\
\text { the caudal fin cut off } \\
\text { Feed without product }\end{array}$ & $\begin{array}{l}\text { Solution of potassium } \\
\text { dichromate } \\
\text { concentrate. } 0.5 \mathrm{mg} / 1 \\
12 \text { zebrafish with the } \\
\text { upper part of the } \\
\text { caudal fin cut off. } \\
\text { Food with the product } \\
\text { " Chigyany ROZ» }\end{array}$ \\
\hline
\end{tabular}

Figure 1. Distribution of Danio rerio fish in the experiment during regeneration of the caudal fin in sewage tap water and in solutions of potassium dichromate with or without the addition of the product "Chigyany ROZ" to the feed

The caudal fin with regenerate was placed in a drop of acridine orange for 5 minutes and enclosed under a cover glass.

The caudal fin of zebrafish was stained according to the generally accepted method (Pirs, 1963) (according to Bartalanffy and Bickins) with a $0.1 \%$ solution of acridine orange diluted with 9 parts of a buffered Krebs-Ringer solution.

Nucleic acid localization sites were detected on the preparations by their fluorescence. Nuclei (DNA) fluoresce in green, and RNA in red and orange.

The area of the regenerating part of the caudal fin was correlated to the lower intact part and calculated by applying a grid on a computer image obtained when shooting with an MBS-10 microscope at a magnification of $10 \times 15$ (see Figure 2).

Statistical processing of the results of the study was carried out according to the Sudent criterion (Platonov, 2001).

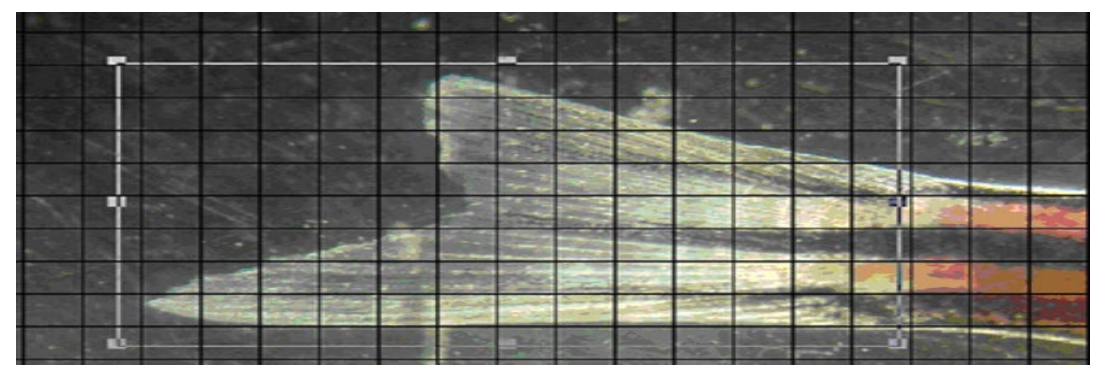

Figure 2. Grid for calculating the area of regeneration of the upper lobe in relation to the area of the lower lobe of the zebrafish caudal fin 
The rate of caudal fin regeneration in zebrafish in the experiment and in the control was determined by the ratio of the areas of the upper regenerating part and the intact lower lobe of the homocercal fin. Figure 3 shows the caudal fins of zebrafish on days 3 and 9 after the upper part was cut off. It was previously shown that complete regeneration of the upper caudal fin lobe in juvenile zebrafish is completed in 14 days (Tian et al., 2019). In this case, the terminal point of research is 9 days, when you can already compare the areas of regenerate in different variants of the experiment (see Figure 3 ).
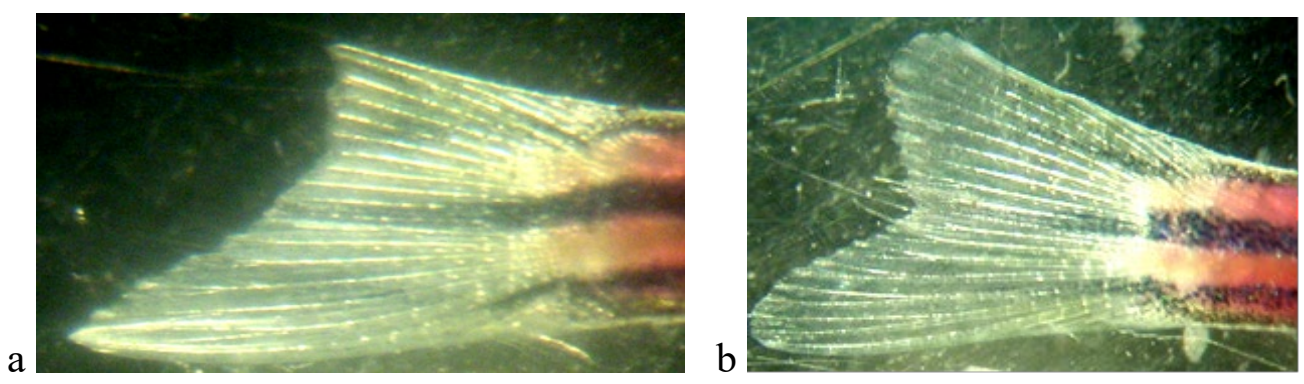

Figure 3. Regeneration of the upper caudal fin blade on days 3 (a) and (9) (control).

The results of the study of regeneration of the upper lobe of the caudal fin in fish treated with Chigyany ROZ on the third day and at the end of the experiment are shown in Figure 3.
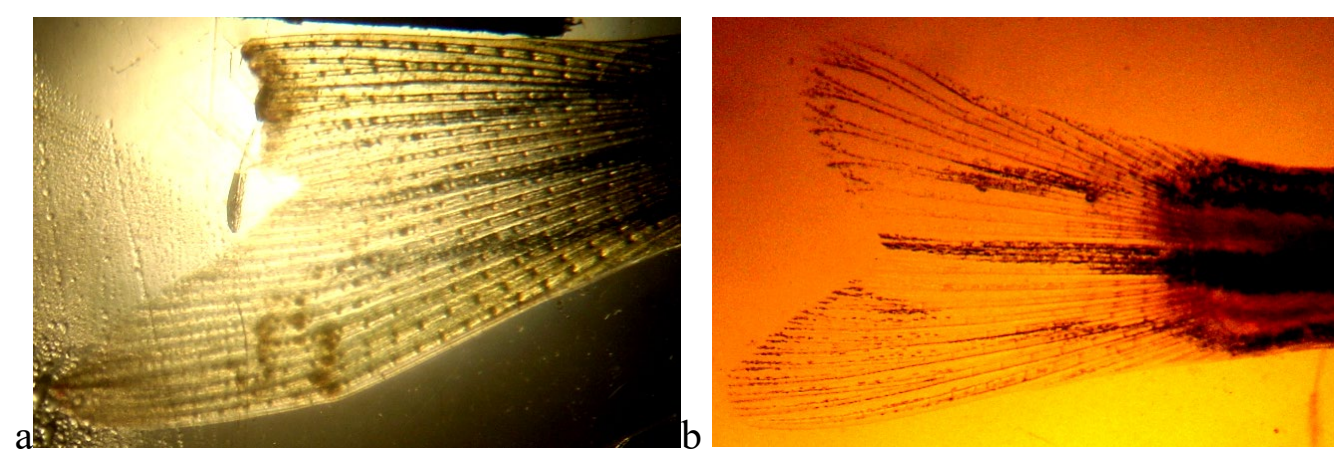

Figure 4. Regeneration of the severed part of the caudal fin in zebrafish fed with the product "Chigyany ROZ" on the 3rd and 9th days of the experiment. The regeneration is almost complete
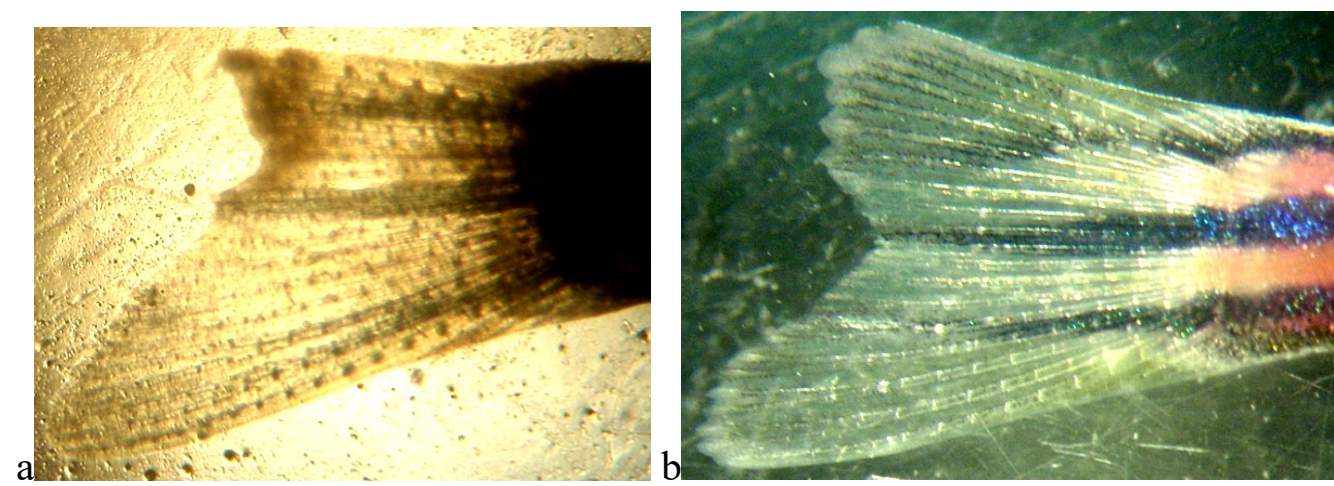

Figure 5. Regeneration of the upper lobe of the caudal fin in fish in a potassium dichromate solution with a concentration of $0.5 \mathrm{mg} / \mathrm{l}$ : a-Day 3; b-day 9 of the experiment (food without the addition of "Chigany ROZ"). Regeneration is only $24 \%$ complete 
As can be seen from Figure 4, the regeneration of the upper lobe of the caudal fin in fish treated with Chigyany ROZ is approximately $40 \%$ faster than in the control. A more accurate calculation of the regeneration rate of the upper fin blade was determined by the ratio of the blastema to the lower fin blade (the results are presented in Table 1).

When fish are found in solutions of potassium dichromate, under the influence of hexavalent chromium, regeneration is suppressed. In a solution of potassium dichromate, regeneration was completed only by $24 \%$, in relation to complete regeneration (Figure 5 ).

In the fourth version of the experiment, the combined effect of potassium dichromate and the product "Chigyany ROZ" coming to fish together with food was studied. This option allows you to answer the main question, whether the product "Chigany ROZ" has a protective property, which acts as a regeneration stimulator.

As in the previous experiments, Figure 6 shows the regenerating caudal fin of zebrafish on Days 3 and 9 after the start of the experiment. In this case, it was possible to establish that the product "Chigyany ROZ" has a protective property and, as it were, removes the negative effect of potassium dichromate on the regeneration process (Table 1).

The table shows such an indicator as the ratio of the regenerating blade to the lower intact blade, which is characteristic of each individual fish studied. In this case, we exclude variational differences associated with fluctuations in the size parameters of individual fish studied.

Figure 6 shows an example of regeneration of the upper lobe of the zebrafish caudal fin under conditions of simultaneous exposure to potassium dichromate solutions and the addition of the product "Chigyany ROZ" to fish feed. Studies have shown that the product "Chigany ROZ" acts as a protector and provides a regeneration process similar to how it occurs in the control. However, the stimulating property of the product "Chigany ROZ" in this case is partially suppressed.
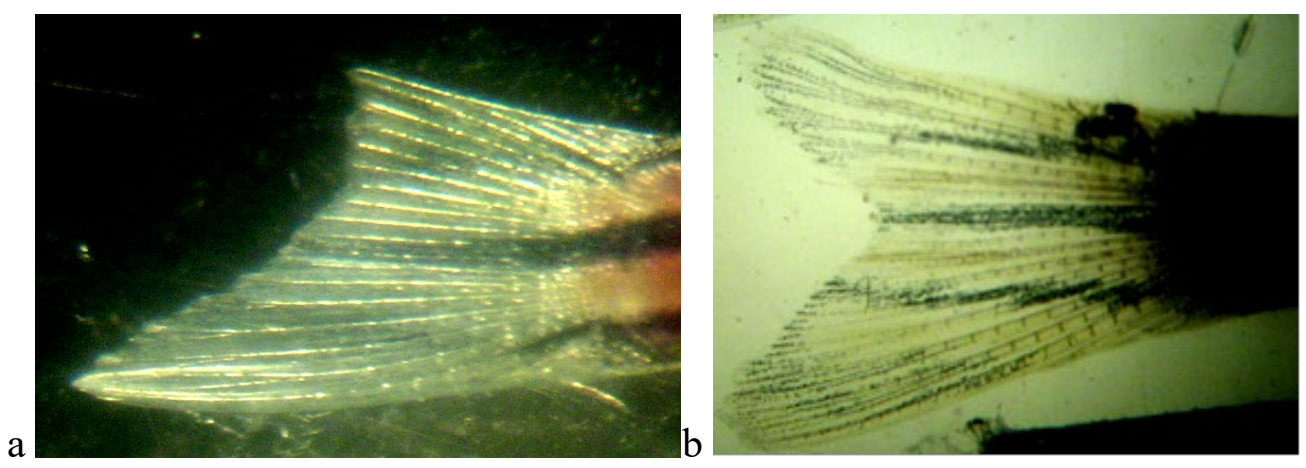

Figure 6. Regeneration of the zebrafish caudal fin blade when exposed to potassium dichromate and when feeding fish with the addition of Danio rerio product. Regeneration occurred in 9 days by $55 \%$

Data on the effect of the studied factors on fin blade regeneration are presented in Table 1.

Table 1. The ratio of the blastema area to the area of the lower fin blade under various conditions of regeneration time $\left(\mathrm{t}_{\mathrm{td}}\right.$-confidence criterion)

\begin{tabular}{cccccc}
\hline Regeneration days & 3-day & 6 - day & td & 9 -day & td \\
\hline With the product "Chigyany ROZ" & $0,17 \pm 0,02$ & $0,43 * \pm 0,05$ & 4,25 & $0,83 * \pm 0,05$ & 5,31
\end{tabular}


With action $\mathrm{K} 2 \mathrm{Withr} 2 \mathrm{O} 7 \mathrm{R} 2 \mathrm{O} 7$ at a concentration of $0.5 \mathrm{mg} / 1$

$\begin{array}{lcccc}0,10 \pm 0,03 & 0,14^{*} \pm 0,06 & 3,46 & 0.24 * \pm 0,04 & 3,04 \\ 0,19 \pm 0.06 & 0,32 \pm 0,03 & 1,84 & 0,55^{*} \pm 0,07 & 2,96 \\ 0.14 \pm 0.06 & 0.28 \pm 0.04 & & 0.43 \pm 0.03 & \end{array}$

Under the influence of potassium

dichromate and "Chigany ROZ"

Control

$0.14 \pm 0.06$

* - significance of the mean difference at $\mathrm{P} \leq 0.05$ (ttst $=2.73$ )

Morphological and histochemical luminescence analysis revealed that the most intensive regeneration of the fin blade occurs on the 9th day of the experiment. In fish that did not take "Chigyany ROZ", the blastema increases by $43 \%$ during this period, and in fish that took "Chigyany ROZ" with food, the blastema increases by up to $83 \%$. in relation to the intact fin blade. Nucleic acids are most intensively detected in regenerates of fish that took "Chigyany ROZ" with food.

Thus, the product "Chigyany ROZ" acts as a stimulator of the regeneration of organs and tissues, but the stimulation of the restoration of a lost organ is not so intense and varies from 12 to $32 \%$ in different periods. The complete regeneration of the upper fin blade is accelerated almost twice.

Thus, the most intensive regeneration process on day 9 was $40 \%$ higher in the fish treated with the product "Chigyany ROZ" compared to the control fish. The rate of regeneration when the product "Chigyany ROZ" is added to fish feed tends to increase the rate of blastema growth compared to the control. Regeneration ends 6 days earlier than in the control, based on the calculation that the complete regeneration of the fin blade takes place in 14 days. Therefore, we can state that the product "Chigyany ROZ" has the property of stimulating the caudal fin regeneration process.

As before, we were able to show that potassium dichromate, as a mutagen and carcinogen, at a concentration of $0.5 \mathrm{mg} / 1$ suppresses the regeneration process of the severed fin blade. For this reason, the combined use of the genotoxicant and the product "Chigyany ROZ" allowed us to identify its protective properties, it protects the regeneration processes. However, the stimulating effect is partially weakened by the toxicant.

To characterize the effect of "Chigyany ROZ" on regeneration processes at the molecular level, we performed histochemical determination of nucleic acids in the upper lobe blastem of the zebrafish caudal fin. For this purpose, we used an intravital luminescent study of the regenerating fin with staining of the preparation with acridine orange. Fin blastema preparations were prepared, taken from control fish, and from fish in various experimental variants described earlier, which underwent regeneration without the use of the product "Chigyany ROZ" and with its use during feeding. Figure 7 shows the regenerating fin preparations in the control on the 3rd day after cutting off the upper lobe (a) and on the ninth day after the start of the experiment (b), when orange tones are visible in the border of the blastema, indicating the accumulation of RNA in the tissues. Perhaps microRNA is synthesized in smaller amounts at this time, and regeneration is accelerated. 

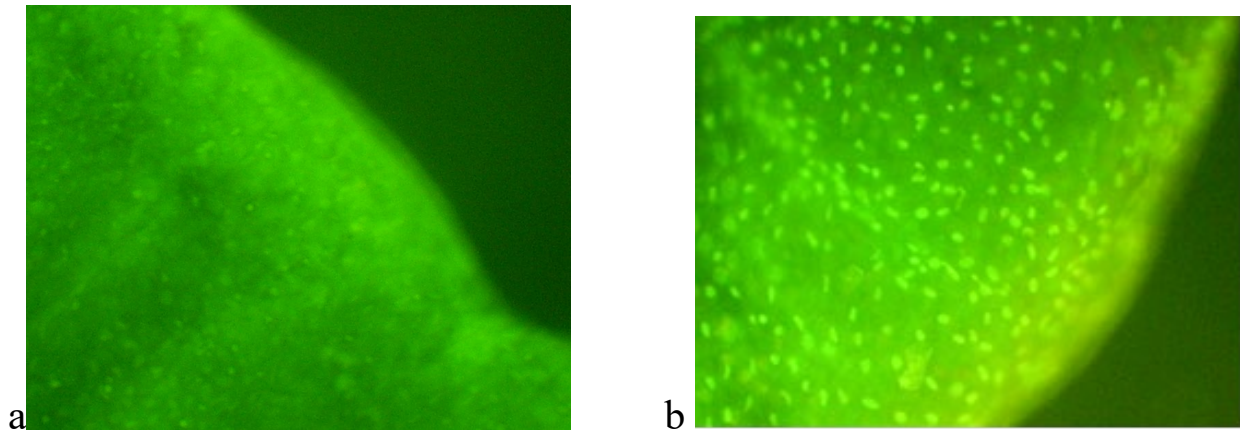

Figure 7. Distribution of nucleic acids in the marginal blastema of the upper regenerating blade of the caudal fin without the use of "Chig-ROZ": a - on the third day of regeneration; b-on the 9 th day of regeneration

Analysis of histochemical preparations shown in Figure 7 shows that on the third day of regeneration in control fish without feeding with "Chigyany ROZ", mainly the DNA of the nuclei is detected, and tissue activation occurs in the area of the fin blade section (green glow increases). At the same time, protein synthesis is only at the initial stages and there is no red glow that reveals RNA. On the ninth day of regeneration, when cell proliferation decreases and cytodifferentiation processes begin, red tones are already noted, indicating an increase in RNA synthesis. This indicates that regeneration is about to be completed (Figure $7 \mathrm{~b}$ ).

On the third day in blastema, green tones are also visible on the preparations of the blastema border, indicating DNA synthesis, in fish that received the product "Chigyany ROZ" with food (Figure 8a). At the same time, red hues. There are almost no signs of RNA accumulation in tissues. Thus, the difference between the regeneration of the caudal fin in zebrafish on the third day without the use of the product "Chigyany ROZ" and with its addition to fish food is not noted. However, in the future, RNA begins to accumulate in regenerating tissues, and by the ninth day, when regeneration with the use of the product "Chigyany ROZ" is almost complete, the greatest accumulation of RNA is noted, as indicated by red fluorescence (Figure 8 b). By this time, regeneration is completed by $83 \%$.
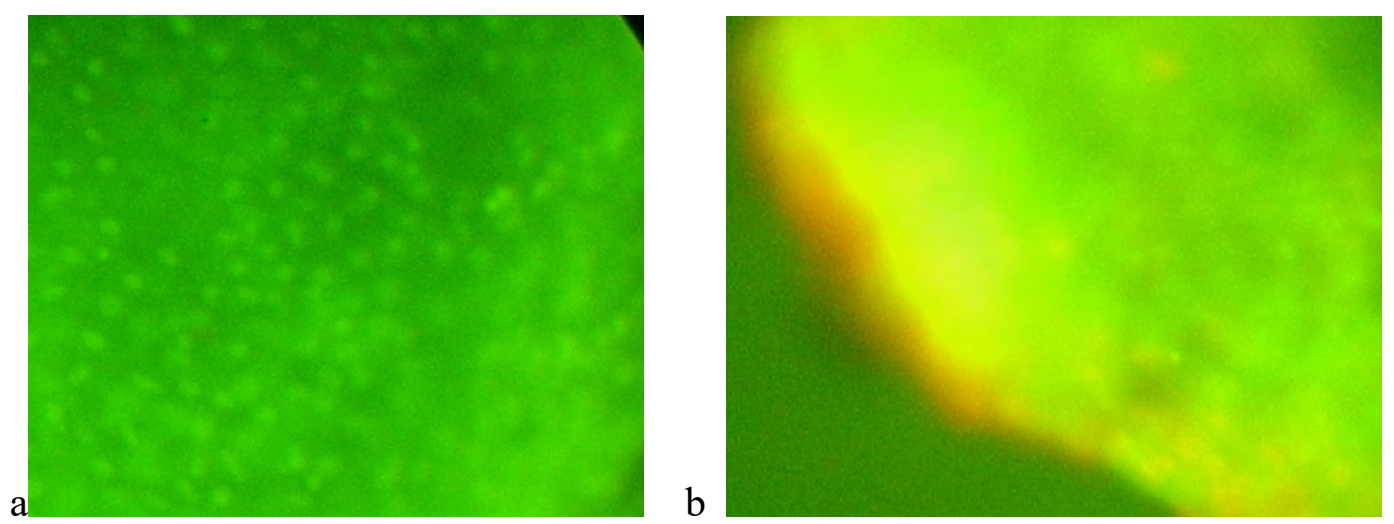

Figure 8. Marginal region of the caudal fin blastema of zebrafish fed with "Chigyany ROZ" food, when stained for the distribution of DNA and RNA (DNA-green fluorescence, RNAorange and red fluorescence); $\mathrm{a}$ - Day 3 of regeneration; b-day 9 of regeneration 
Figure 9 shows preparations of blastema of the regenerating caudal fin blade in fish in a potassium dichromate solution with a concentration of $0.5 \mathrm{mg} / \mathrm{l}$. At the same time, zebrafish were not fed the product "Chigyany ROZ".
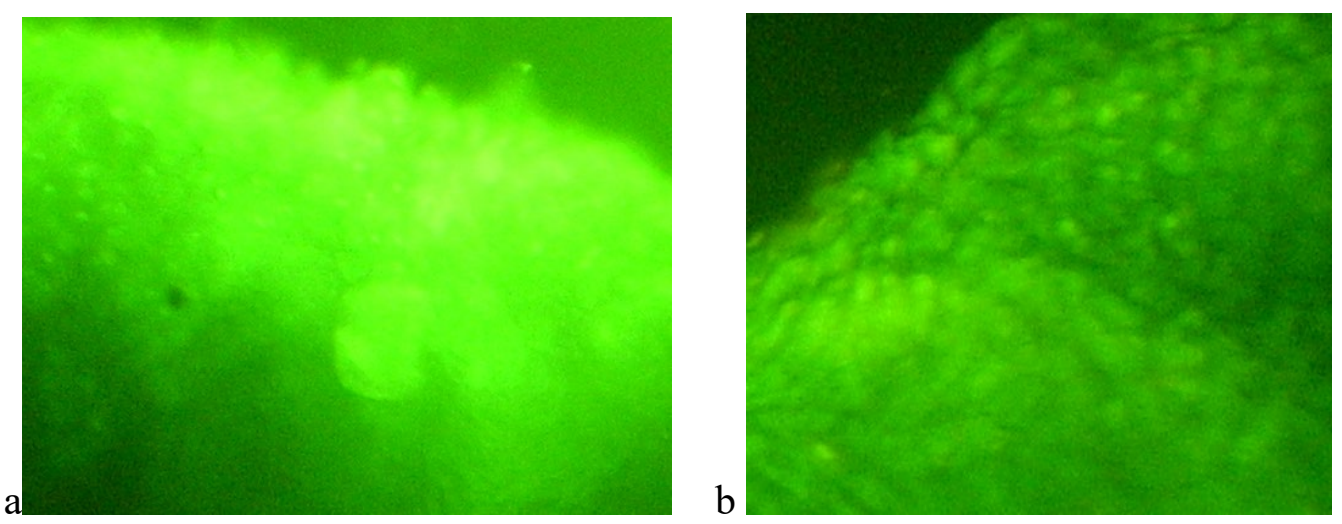

Figure 9. Distribution of nucleic acids in the blastem of the regenerating fin of fish in potassium dichromate solutions: a - 3rd day of regeneration; b-9th day of regeneration

When potassium dichromate acts on the regeneration process, the amount of RNA practically does not increase for 9 days. Regeneration is suppressed under the influence of a genotoxicant, which is hexavalent chromium.

With the simultaneous action of potassium dichromate and the product "Chigyany ROZ", which comes in the form of an additive to fish feed, the process of accumulation of nucleic acids in blastema tissues is similar to the control. However, the stimulating effect of the product "Chigany ROZ" is not observed. It seems that the reaction of the product "Chigyany ROZ" is aimed at protecting the regeneration processes. The picture of the effect of potassium dichromate and the product "Chigyany ROZ" on the distribution of nucleic acids in blastema tissues on the 3rd and 9th days of regeneration is shown in Figure 10.
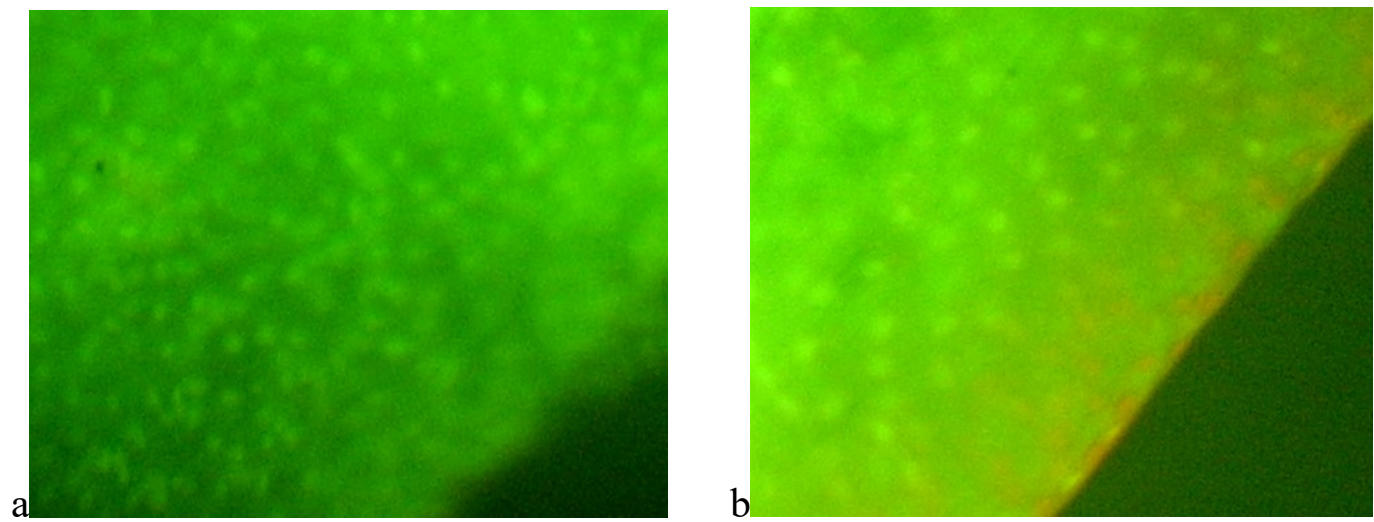

Figure 10. Distribution of nucleic acids in the edge of the zebrafish regenerating fin blastema after combined exposure to potassium dichromate and fish consumption of feed with the product "Chigyany ROZ": $a$ - three days after the start of the experiment; $b-9$ days after the start of the experiment 
During the most intensive regeneration on day 9, the difference between control and experience is most pronounced. However, the consumption of the product "Chigyany ROZ" with food, fish, which are in solutions of genotoxicant leads to the fact that a red glow is visible in the border, indicating the accumulation of RNA in the blastem. It can be assumed that using luminescence microscopy, microRNAs are also detected among RNAs. The preparation shows nuclei with DNA (green) and areas of the blade where RNA is localized (red and orange).

\section{Findings}

The stimulating and protective properties of the product "Chigyany ROZ" were tested on juvenile aquarium fish-Danio rerio, which has an amazing ability to regenerate various tissues and organs, including the regeneration of the heart (Jopling et al., 2010; Mersereau et al., 2015; Poss, 2002). Much of the research has focused on fish caudal fin regeneration (Azevedo et al., 2011). Zebrafish caudal fin regeneration is a very convenient model for solving many problems related to cell differentiation, pharmacology, and aquatic toxicology (Bambino \& Chu, 2017). In previous studies of fin regeneration, the upper and lower lobes were amputated. To quantify the rate of caudal fin regeneration, this is not very convenient for biometric processing of the results obtained, since the area of the fin blades in different zebrafish can vary greatly. But the ratio of blade areas remains constant. To exclude these fluctuations, we cut off only the upper blade in this study, and the regeneration rate under the action of the toxicant and the product "Chigyany ROZ" was estimated by the ratio of the areas of the regenerating upper blade and the lower intact blade. A similar technique was used earlier in previous studies on the effect of interleukins on caudal fin regeneration in zebrafish (Simakov et al., 2020).

Half of the fish received granulated feed with the product "Chigyany ROZ", and the other half without "Chigyany ROZ". Dosage of "Chigyany ROZ" per day-0.5 tablets per $200 \mathrm{~g}$ of live weight of fish. The dynamics of the regeneration study was performed on days 3, 6 and 9. On day 3 and 9, vital preparations were prepared from regenerating fins with DNA and RNA staining in acridine orange and subsequent examination under a luminescent microscope.

It was found that the most intensive regeneration of the fin blade occurs on the 9th day of the experiment. In fish in clean water that did not take the product "Chigyany ROZ", blastema increases by $43 \%$, while in fish that took the product "Chigyany ROZ" with food, blastema increases by $83 \%$. On the preparations, DNA fluoresces green, and the areas where RNA is localized are colored in orange and red tones. RNA is most intensively detected in regenerates of fish that have taken the product "Chigyany ROZ" with food, both in aquariums with clean tap water, and in aquariums with clean tap water. present in solutions of potassium dichromate.

In fish that are in solutions of potassium dichromate, but did not receive the addition of the test product, regeneration is suppressed and the fin blade is restored only by $24 \%$ in 9 days. At the same time, histochemical preparations did not show an increase in RNA on the ninth day. When the product "Chigyany ROZ" is added to the granulated fish feed contained in the genotoxicant solution, regeneration of the caudal fin blade is restored, but the stimulating effect of the product is reduced, and regeneration is completed only by $55 \%$ in 9 days. RNA fluorescence detected by acridine orange in the blastema rim 
after fish ingestion of the product "Chigyany ROZ". it is most intensively manifested in the combined action of potassium dichromate and the studied dairy product.

Thus, the product "Chigyany ROZ" acts as a stimulator of regeneration of organs and tissues. It also protects regeneration from being suppressed by the genotoxicant. In the latter case, the regenerationstimulating properties of the product are somewhat reduced. Therefore, the product "Chigyany ROZ" can be recommended for accelerating regeneration processes, and for protecting regeneration processes under the action of mutagenic and carcinogenic substances on the body. All the established regularities of genotoxicants and the product "Chigyany ROZ" can most likely be manifested in the regeneration of organs and tissues in other vertebrates and humans, this is proved by many works indicating the identity of genetic processes occurring during regeneration in higher and lower vertebrates (Howe et al., 2013; Lin et al., 2016; Ribas \& Piferrer, 2014).

\section{Conclusion}

Analysis of the obtained data allows us to conclude that the product "Chigany ROZ" has protective properties and protects the regenerating organ from the effects of the genotoxicant-potassium dichromate.

The regeneration rate, judging by the ratio of the regenerate area and the lower intact caudal fin blade, is slightly higher. when adding the product "Chigyany ROZ" to the feed. Regeneration by the 9th day of the experiment after amputation of the fin blade is completed by $83 \%$, while in the control, the regeneration rate in fish is almost two times smaller in area.

In potassium dichromate solutions without the test product, regeneration of the upper caudal fin blade is significantly suppressed (it is only $24 \%$ of the lower control area of the caudal fin). The addition of the lactic acid product "Chigyany ROZ" to the feed stimulates the synthesis of RNA in the regenerate blastem both in pure water and in a solution of potassium dichromate with a concentration of $0.5 \mathrm{mg} / \mathrm{l}$.

\section{References}

Aleksandrov, A. N., \& Simakov, Y. G. (2013). Ispol'zovanie regeneratsii khvudovogo finnika u Brachy Danio rerio v biotestirovanii vodnoy sredy [Use of tail fin regeneration in BrachyDanio rerio in water environment biotesting]. Abstracts of the International conference Moscow, February 4-6, 2013. Binom. Laboratory of Knowledge.

Azevedo, A. S., Grotek, B., Jacinto, A., Weidinger, G., \& Saúde, L. (2011). The regenerative capacity of the zebrafish caudal fin is not affected by repeated amputations. PLoS One, 6(7), e22820. https://doi.org/10.1371/journal.pone.0022820

Bambino, K., \& Chu, J. (2017). Zebrafish in Toxicology and Environmental Health. Curr TopDev Biol., 124, 331-367. https://doi.org/10.1016/bs.ctdb.2016.10.007

Belyaeva, N. F., Kashirtseva, V. N., \& Medvedeva, N. V. (2010). Zebrafish as a model in biomedical research. Biomed. Khim, 56(1), 120-131. https://doi.org/10.18097/pbmc20105601120

Bradford, Y. M., Toro, S., Ramachandran, S., Ruzicka, L., Howe, D. G., Eagle, A., Kalita, P., Martin, R., Taylor Moxon, S. A., Schaper, K., \& Westerfield, M. (2017). Zebrafish models of human disease: gaining insight into human disease at ZFIN. ILAR journal, 58(1), 4-16. https://doi.org/10.1093/ilar/ilw040

González-Rosa, J. M., Burns, C. E., \& Burns, C. G. (2017). Zebrafish heart regeneration: 15 years of discoveries. Regeneration, 4(3), 105-123. https://doi.org/10.1002/reg2.83

Howe, K., Clark, M. D., Torroja, C. F., Torrance, J., Berthelot, C., Muffato, M., Collins, J. E., Humphray, S., McLaren, K., Matthews, L., McLaren, S., Sealy, I., Caccamo, M., Churcher, C., Scott, C., 
Barrett, J. C., Koch, R., Rauch, G. J., White, S., Chow, W., ... Teucke, M. (2013). The zebrafish reference genome sequence and its relationship to the human genome. Nature, 496(7446), 498503. https://doi.org/10.1038/nature12111

Ishida, T., Nakajima, T., Kudo, A., \& Kawakami, A. (2010). Phosphorylation of Junb family proteins by the Jun N-terminal kinase supports tissue regeneration in zebrafish. Dev Biol., 340, 468-479. https://doi.org/10.1016/j.ydbio.2010.01.036

Jopling, C., Sleep, E., Raya, M., Martí, M., Raya, A., \& Belmonte, J. C. I. (2010). Zebrafish heart regeneration occurs by cardiomyocyte dedifferentiation and proliferation. Nature, 464(7288), 606609. https://doi.org/10.1038/nature08899

Kachanov, D. A., Bardakov, M. O., Lukin, E. N., Mkrtchjan, G. V., Gazdanova, N. V., Akopjan, K. A., Evstratova, A. V., Badalyants, D. A., Khachatryan, A. V., \& Shahnazarjan, R. L. (2020). Comparative review of the possibilities of using Danio rerio (zebrafish) as a model object in preclinical studies. International research journal, 6(96), 107-111. https://researchjournal.org/medical/sravnitelnyj-obzor-vozmozhnostej-ispolzovaniya-danio-rerio-zebrafish-vkachestve-modelnogo-obekta-v-doklinicheskix-issledovaniyax/

Karnaukhov, V. N. (2002). Lyuministsentnyi analiz kletok [Luminescent cell analysis]. Pushchino.

Kettleborough, R. N., Busch-Nentwich, E. M., Harvey, S. A., Dooley, C. M., de Bruijn, E., van Eeden, F., Sealy, I., White, R. J., Herd, C., Nijman, I. J., Fényes, F., Mehroke, S., Scahill, C., Gibbons, R., Wali, N., Carruthers, S., Hall, A., Yen, J., Cuppen, E., \& Stemple, D. L. (2013). A systematic genome-wide analysis of zebrafish protein-coding gene function. Nature, 496(7446), 494-497. https://doi.org/10.1038/nature11992

Lin, J., Zhang, W., Zhao, J. J., Kwart, A. H., Yang, C., Ma, D., Ren, X., Tai, Y. T., Anderson, K. C., Handin, R. I., \& Munshi, N. C. (2016). A clinically relevant in vivo zebrafish model of human multiple myeloma to study preclinical therapeutic efficacy. Blood, The Journal of the American Society of Hematology, 128(2), 249-252. https://doi.org/10.1182/blood-2016-03-704460

Mersereau, E. J., Poitra, S. L., Espinoza, A., Crossley, D. A., 2nd, \& Darland, T. (2015). The effects of cocaine on heart rate and electrocardiogram in zebrafish (Danio rerio). Comparative biochemistry and physiology. Toxicology \& pharmacology: CBP, 172-173, 1-6. https://doi.org/10.1016/j.cbpc.2015.03.007

Niethammer, P., Grabher, C., Look, A. T., \& Mitchison, T. J. (2009). A tissue-scale gradient of hydrogen peroxide mediates rapid wound detection in zebrafish. Cell Death Differ, 1, 431-442. https://doi.org/10.1038/nature08119

Nikiforov-Nikishin, D. L., Kochetkov, N. I., Smorodinskaya, S. V., Tatarenko, P. Y., \& Matveeva, D. M. (2021, September). Toxicity of metal chelates mixture in aquatic environment at Danio rerio. In IOP Conference Series: Earth and Environmental Science (Vol. 839, No. 5, p. 052010). IOP Publishing. https://doi.org/10.1088/1755-1315/839/5/052010

Pirs, E. (1963). Histochemistry. ILL.

Platonov, A. E. (2001). Statisticheskii analiz v meditsine $i$ biologii [Statistical analysis in medicine and biology]. RAMN.

Poss, K. D. (2002). Heart Regeneration in Zebrafish. Science, 298, 2188-2190. https://doi.org/10.1126/science.1077857

Ribas, L., \& Piferrer, F. (2014). The zebrafish (Danio rerio) as a model organism, with emphasis on applications for finfish aquaculture research. Rev Aquacult, 6, 209-240. https://doi.org/10.1111/raq.12041

Simakov, Y. G., Purtskhvanidze, V. A., Golovacheva, N. A., Bychkova, L. I., \& Omelchuk, N. N. (2020). Stimulation of erythropoeisis Regenerative processes in the Danio rerio fish under the influence of interleukin-2. International Journal of Advanced Research in Engineering and Technology (IJARET), 11(5), 309-317.

Smorodinskaya, S. V., \& Simaov, Y. G. (2019). Changes in the DNA structure and morphology of Danio rerio erythrocyte nuclei during water contamination with potassium dichromate. Food 2035. Biophysics and technology. Collection of materials of the scientific and practical conference. "Yulis". With. 
Tian, S., Teng, M., Meng, Z., Yan, S., Jia, M., Li, R., Liu, L., Yan, J., Zhou, Z., \& Zhu, W. (2019). Toxicity effects in zebrafish embryos (Danio rerio) induced by prothioconazole. Environ Pollut. 255(2), 113269. https://doi.org/10.1016/j.envpol.2019.113269

Traver, D., Winterer, A., Stern, H. M., Mayhall, E. A., Langenau, D. M., Kutok, J. L., Look, A. T., \& Zon, L. I. (2004). Effects of lethal irradiation in zebrafish and rescue by hematopoietic cell transplantation. Blood, 104(5), 1298-1305. https://doi.org/10.1182/blood-2004-01-0100 